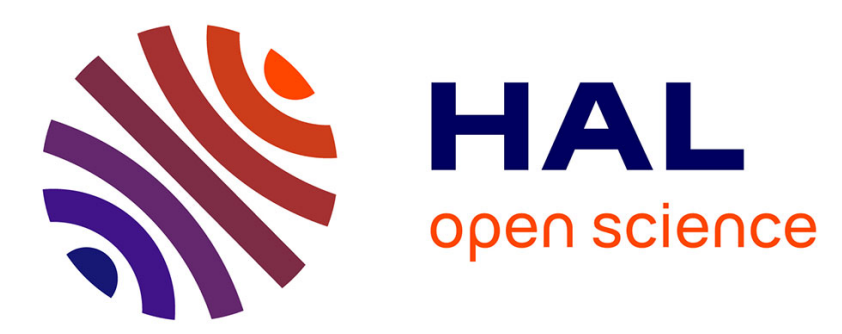

\title{
A novel approach for ontological representation of analytic hierarchy process
}

Yongxin Liao, Eduardo Rocha Loures, Osiris Junior Canciglieri, Hervé Panetto

\section{To cite this version:}

Yongxin Liao, Eduardo Rocha Loures, Osiris Junior Canciglieri, Hervé Panetto. A novel approach for ontological representation of analytic hierarchy process. Advanced Materials Research, 2014, 945-949, pp.675-682. 10.4028/www.scientific.net/AMR.988.675 . hal-01044953

\section{HAL Id: hal-01044953 https://hal.science/hal-01044953}

Submitted on 24 Jul 2014

HAL is a multi-disciplinary open access archive for the deposit and dissemination of scientific research documents, whether they are published or not. The documents may come from teaching and research institutions in France or abroad, or from public or private research centers.
L'archive ouverte pluridisciplinaire HAL, est destinée au dépôt et à la diffusion de documents scientifiques de niveau recherche, publiés ou non, émanant des établissements d'enseignement et de recherche français ou étrangers, des laboratoires publics ou privés. 


\title{
A Novel Approach for Ontological Representation of Analytic Hierarchy Process
}

\author{
Yongxin Liao ${ }^{1, a}$, Eduardo Rocha Loures ${ }^{1, b}$, Osiris Canciglieri Junior ${ }^{1, c}$ and \\ Hervé Panetto ${ }^{2,3, d}$
}

${ }^{1}$ Pontifical Catholic University of Paraná, PPGEPS, Rua Imaculada Conceição, 1155, Curitiba, Paraná, Brazil.

2 Université de Lorraine, CRAN, UMR 7039, Boulevard des Aiguillettes B.P.70239, 54506

Vandoeuvre-lès-Nancy, France; ${ }^{3}$ CNRS, CRAN, UMR 7039, France.

ayongxin.liao@pucpr.br, beduardo.loures@pucpr.br, 'osiris.canciglieri@pucpr.br, dherve.panetto@univ-lorraine.fr

Keywords: Decision Making, Analytic Hierarchy Process, Ontology, Reasoning Rule.

\begin{abstract}
The Analytic Hierarchy Process (AHP) is one of the widely accepted and frequently used priority assessment methods. A number of studies, which employed the AHP method, have already been carried out in different domains to support their multi-criteria decision-making definitions and applications. However, few researchers have paid attentions to extract and represent the important concepts and their relationships from the AHP method itself. The aim of this study is firstly to propose a way for representing the AHP method as an AHP Ontology together with a set of reasoning rules. Then, a prototype assessment tool is developed to show the possibility of obtaining more flexibility and reusability of this ontological representation.
\end{abstract}

\section{Introduction}

The Analytic Hierarchy Process, which is proposed by Saaty [1] since the 1970s, is one of the widely accepted and frequently used mathematic analysis method that supports multi-criteria decision-making. A significant number of research projects employed the AHP method to support decision-making in their works and have already been benefited from it [2]. Meanwhile, keeping pace with these increasing needs, the development of decision-making tools based on the AHP method has attracted many attentions of some software companies, such as, Decision Lens ${ }^{1}$, Expert Choice ${ }^{2}$, and Logical Decision ${ }^{3}$. However, among these research projects and implemented toolkits, little work has been done in specifying and formalizing the important concepts together with their relationships from the AHP method itself.

Recently, this existing issue is noticed by some researchers, who began to apply a kind of incomplete ontological representation of the AHP method in their decision-making approaches. For example, Kornyshova and Deneckère [3] proposed a Decision Making Ontology, which aims to formalize the concepts and their relationships inside the knowledge that related to decision-making procedures and the AHP method, for supporting the decision-making during the information systems engineering. Tran, et al. [4] developed a QoS ontology to describe the information about Web Services in detail and a QoS-based algorithm that adopted the AHP method as the evaluation mechanism to assist Web Services ranking. Wasielewska, et al. [5] presented a domain ontology, which captures the concepts that are needed by an AHP algorithm, for assisting an user in specifying required resources to execute a job and then to evaluate the contract proposals based on the those resources and their comparisons. Although efforts have been made by these researches, several existing drawbacks still can be found as follows: (i) Some researches only propose a class hierarchy in an ontology to capture the concepts from the AHP method, but without specifying the relationships

\footnotetext{
${ }^{1}$ Decision Lens: http://www.decisionlens.com/

${ }^{2}$ Expert Choice: http://expertchoice.com/

${ }^{3}$ Logical Decision: http://www.logicaldecisions.com/
} 
between these concepts; (ii) There exist some researches, which formalized both concepts and their relationships from the AHP method. However, the evaluation mechanisms in the AHP method are always designed as part of fixed algorithms that are embedded inside priority assessment tools; (iii) The ontological representation of the AHP method is usually mixed with the information that are needed to be assessed, which lacks of flexibility and reusability. Therefore, there remain needs for an approach to address these limitations.

The purpose of this paper is to propose a way to ontologically represent the AHP method, which not only specifies the concepts and their relationships that are necessary for applying the AHP method, but also realizes the priority assessment and consistency evaluation mechanisms in the AHP method through corresponding reasoning rules. In the remains of this paper, a brief introduction of the AHP method is firstly given to describe the background of our proposition. Then, the proposed ontological representation is presented to highlight our contribution. After that, a priority assessment prototype, based on the ontological representation, is used to show the possibility of obtaining more flexibility and reusability. At the end, we conclude this paper and discuss on future works.

\section{The Analytic Hierarchy Process (AHP)}

The priority assessment in the AHP method is based on the mathematical analysis of a set of pairwise comparisons, in which, the priority scales between each two related terms are relying on the professional judgments of domain experts [6]. Of course, these human judgments may not be always consistent. The AHP method also provides a theory of consistency evaluation to assist experts to improve their judgments and to obtain better consistency [7]. In general, the process of applying the AHP method can be divided into the following five steps:

(1) Define a problem area and determine a goal (the top level) of the decision-making.

(2) Select a set of evaluation criteria (the intermediate level) and a set of alternatives (the lowest level) to structure a decision hierarchy. An example of this hierarchy can be seen in Fig. 1.

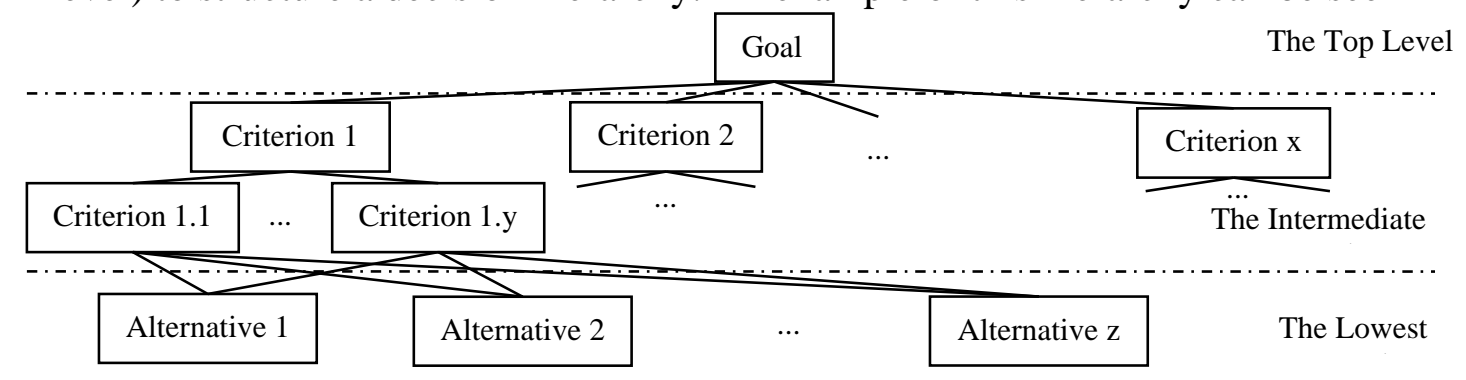

Fig. 1. An Example of the Decision Hierarchy

(3) Generate a set of pairwise comparison matrices, in which, all compared terms in a matrix are in the same level of the decision hierarchy and all comparisons in a matrix are with respect to a related term from upper level. Let term $_{1}, \ldots$, term $n$ be the set of criteria or alternatives in a matrix and the set of comparison weights between each two terms are $w_{11}, \ldots, w_{n n}$ that are

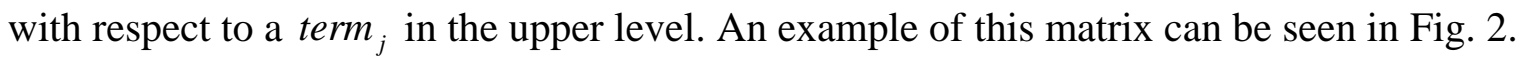

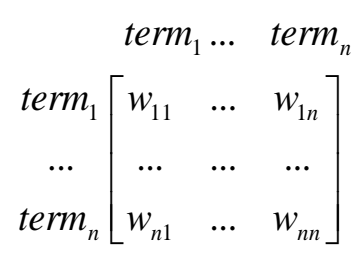

Fig. 2. An Example of the Comparison Matrix

(4) Calculate the global priority of each criterion in the decision hierarchy and use them to weight the final priority of each alternative. In this paper, we only introduce one way to calculate the priority of each term in a matrix, which can be decomposed into five sub-steps: 
a. Sum up the weights in each column in a matrix. Let $c_{i}$ be the sum value of the column $i$, where $i=1, \ldots, n$. Then $c_{i}=\sum_{x=1}^{n} w_{x i}$.

b. Divide the weight of each comparison pair by the sum value of its column. Let $q_{j i}$ be the quotient at the raw $j$ and the column $i$ of that matrix, where $i=1, \ldots, n$ and $j=1, \ldots, n$. Then $q_{j i}=w_{j i} / c_{i}$

c. Calculate the average values in each row to obtain the priority of the term in that row. Let $p_{y}$ be the priority of term $_{y}$, where $y=1, \ldots, n$, then $p_{y}=\frac{1}{n}\left(\sum_{z=1}^{n} q_{y z}\right)$.

d. The global priority of a criterion is obtained from its temporary priority in the matrix multiplied by the global priority of the term in its upper level that the matrix respects.

e. The final priority of an alternative is the sum of all its temporary priorities in the related matrices multiplied by the global priority of the corresponding criterion.

(5) Evaluate the consistency of the priority assessment. This checking process can be decomposed into three sub-steps:

a. Calculate the Principal Eigenvalue (PE) of a matrix. Let $\lambda_{\max }$ be the PE of the matrix, then $\lambda_{\max }=\sum_{z=1}^{n} c_{z} * p_{z}$.

b. Calculate the Consistency Index (CI) of a matrix. Let $C I$ be the CI of the matrix, then $C I=\frac{\lambda_{\max }-n}{n-1}$.

c. Calculate the Consistency Ratio (CR) of a matrix based on the Random Consistency Index (RI, as shown in Table 1). If the CR $<10 \%$, this measurement is consistent and acceptable. Let $C R$ be the CR of the matrix, then $C R=C I / R I$

Table 1. Part of The Random Consistency Indexes [8]

\begin{tabular}{|c|c|c|c|c|c|c|c|c|c|c|}
\hline $\mathrm{n}$ & 1 & 2 & 3 & 4 & 5 & 6 & 7 & 8 & 9 & 10 \\
\hline $\mathrm{RI}$ & 0 & 0 & 0.58 & 0.9 & 1.12 & 1.24 & 1.32 & 1.41 & 1.45 & 1.49 \\
\hline
\end{tabular}

\section{The Ontological Representation of the AHP Method}

Based on the above-introduced AHP method, an ontological representation is proposed to deal with the three existing drawbacks that we mentioned in the first section. In order to use an existing reasoning engine to assist the creation of the decision hierarchy and the calculation process, the ontological representation is in line with the OWL2 ${ }^{4}$ standard and results in an ontology, which is named AHP Ontology. An overview of the main structure of this ontology is presented in Fig. 3.

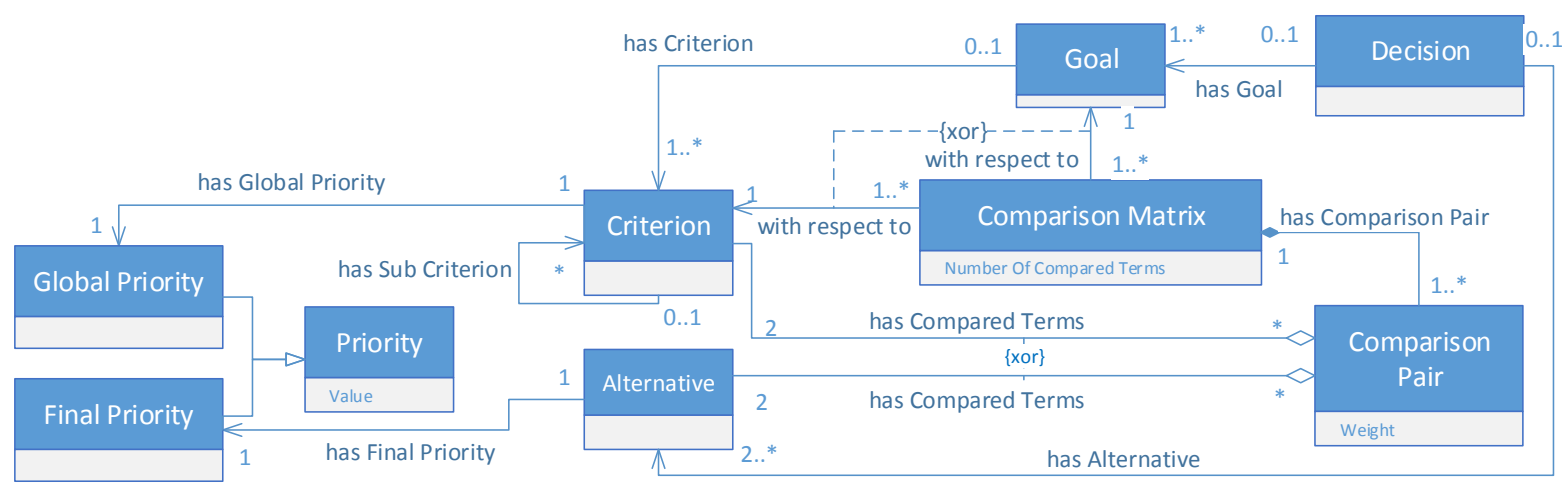

Fig. 3. An Overview of the Main Structure of the AHP Ontology

${ }^{4}$ OWL 2: http://www.w3.org/TR/owl2-overview/ 
To be more specific, following the ontology creation guideline that is presented in [9], the AHP Ontology is introduced with more details in the remains of this section.

Domain Determination. The scope of the AHP Ontology is defined as the decision-making domain. For the step (1), (2), and (3) of the AHP method, which we mentioned in previous section, this ontology is supposed to use appropriate Classes, Properties, and Individuals to represent the main concepts and their relationships. Meanwhile, for the step (4) and (5), this ontology should also contain a number of reasoning rules that can perform priority assessment and consistency evaluation.

Define Classes and Class Hierarchy. As can be seen from Table 2, nine main classes together with their descriptions are listed.

Table 2. The Classes and Their Descriptions

\begin{tabular}{|c|c|c|}
\hline \multicolumn{2}{|c|}{ Classes } & Descriptions \\
\hline \multicolumn{2}{|c|}{ Decision } & The judgment of selecting an alternative for an objective. \\
\hline \multicolumn{2}{|c|}{ Goal } & The objective that a decision making intends to achieve. \\
\hline \multicolumn{2}{|c|}{ Alternative } & The available candidate that can be chosen during the decision-making. \\
\hline \multicolumn{2}{|c|}{ Criterion } & The rule or principle for comparing something. \\
\hline \multirow[t]{2}{*}{ Priority } & GlobalPriority & The indicator that shows the importance of a Criterion over others. \\
\hline & FinalPriority & The indicator that shows the importance of an Alternative over others. \\
\hline \multicolumn{2}{|c|}{ ComparisonPair } & The pair that compares two terms with respect to a Criterion or a Goal. \\
\hline \multicolumn{2}{|c|}{ ComparisonMatrix } & The matrix that is composed of a number of Comparison Pairs. \\
\hline
\end{tabular}

Define Properties and Property Restrictions. As can be seen from Table 3 and Table 4, ten object properties and five data properties together with their restrictions are listed.

Table 3. Object Properties and Their Restrictions

\begin{tabular}{|c|c|}
\hline Object Properties & Descriptions (D) and Property Restrictions (R) \\
\hline hasGoal & $\begin{array}{l}\text { D: Each Decision has one or more Goal as its objectives. } \\
\text { R: Decision hasGoal } \min 1 \text { Goal }\end{array}$ \\
\hline hasAlternative & $\begin{array}{l}\text { D: Each Decision Making has at least two Alternatives to be selected. } \\
\text { R: Decision hasAlternative } \min 2 \text { Alternative }\end{array}$ \\
\hline hasCriterion & $\begin{array}{l}\text { D: Each Goal has one or more Criterion for the priority assessment. } \\
\text { R: Goal hasCriterion } \min 1 \text { Criterion }\end{array}$ \\
\hline hasSubCriterion & $\begin{array}{l}\text { D: Each Criterion can be decomposed into zero or more sub Criteria. } \\
\text { R: Criterion hasSubCriterion some Criterion }\end{array}$ \\
\hline hasFinalPriority & $\begin{array}{l}\text { D: Each Alternative has exactly } 1 \text { Final Priority as the result of a decision-making } \\
\text { R: Alternative hasFinalPriority exactly } 1 \text { FinalPriority }\end{array}$ \\
\hline hasGlobalPriority & $\begin{array}{l}\text { D: Each Criterion has exactly } 1 \text { Global Priority with respect to Goal } \\
\text { R: Criterion hasGlobalPriority exactly } 1 \text { GlobalPriority }\end{array}$ \\
\hline hasComparisonPair & $\begin{array}{l}\text { D: Each Comparison Matrix has one or more Comparison Pairs } \\
\text { R: ComparisonMatrix hasComparisonPair } \min 1 \text { ComparisonPair }\end{array}$ \\
\hline withRespectTo & $\begin{array}{l}\text { D: Each Comparison Matrix is with respect to exactly one Criterion or Goal } \\
\text { R: ComparisonMatrix withRespectTo exactly } 1 \text { (Criterion or Goal) }\end{array}$ \\
\hline hasFirstTerm & $\begin{array}{l}\text { D: Each Pair of Comparison contains two terms that compare to each other. } \\
\text { It has exactly one Alternative or Criterion as the first term. } \\
\text { R: ComparisonPair hasFirstTerm exactly } 1 \text { (Alternative or Criterion) }\end{array}$ \\
\hline hasSecondTerm & $\begin{array}{l}\text { D: Each Pair of Comparison contains two terms that compare to each other. } \\
\text { It has exactly one Alternative or Criterion as the second term. } \\
\text { R: ComparisonPair hasSecondTerm exactly } 1 \text { (Alternative or Criterion) }\end{array}$ \\
\hline
\end{tabular}


Table 4. The Data Properties and Their Restrictions

\begin{tabular}{|l|l|}
\hline \multicolumn{1}{|c|}{ Data Properties } & \multicolumn{1}{c|}{ Descriptions (D) and Property Restrictions (R) } \\
\hline hasValue & $\begin{array}{l}\text { D: Each Priority has exactly 1 Value as the indictor of importance. } \\
\text { R: Priority hasValue exactly 1 double }\end{array}$ \\
\hline $\begin{array}{l}\text { hasNumberOfCompared } \\
\text {-Terms }\end{array}$ & $\begin{array}{l}\text { D: Each Matrix has exactly 1 Number to show the size of that matrix, } \\
\text { namely, how many terms are compared to each other in that matrix. } \\
\text { R: ComparisonMatrix HasNumberOfComparedTerms exactly } 1 \text { integer }\end{array}$ \\
\hline hasI & $\begin{array}{l}\text { D: Each Comparison Pair has exactly 1 Number to show which column it is } \\
\text { located in a matrix } \\
\text { R: ComparisonPair hasI exactly 1 integer }\end{array}$ \\
\hline hasJ & $\begin{array}{l}\text { D: Each Comparison Pair has exactly 1 Number to show which row it is } \\
\text { located in a matrix } \\
\text { R: ComparisonPair hasJ exactly } 1 \text { integer }\end{array}$ \\
\hline hasWeight & $\begin{array}{l}\text { D: Each Comparison Pair has exactly 1 Weight, which is the comparison of } \\
\text { importance from its Frist Term to its Second Term } \\
\text { R: ComparisonPair hasWeight exactly } 1 \text { double }\end{array}$ \\
\hline
\end{tabular}

Create Reasoning Rules for the Calculation. In order to perform the priority assessment and consistency evaluation through a reasoning engine, a number of Properties are added to support the creation of reasoning rules. Limited by the space, in this paper, only a small part of the reasoning rules that are used to calculate a $3 \times 3$ matrix is presented as an example. Table 5 and Table 6 list one additional object property and ten additional data properties together with their descriptions and restrictions for the example.

Table 5. The Additional Object Property for a $3 \times 3$ matrix

\begin{tabular}{|c|l|}
\hline Object Property & \multicolumn{1}{c|}{ Description (D) and Property Restriction (R) } \\
\hline hasSuggestedAlternative & $\begin{array}{l}\text { D: Each Decision has one or more alternatives as suggested selections } \\
\text { R: Decision hasSuggestedAlternative min 1 Alternative }\end{array}$ \\
\hline
\end{tabular}

Table 6. The Additional Data Properties for a $3 \times 3$ matrix

\begin{tabular}{|c|c|}
\hline Data Properties & $\begin{array}{c}\text { Descriptions (D) and Property Restrictions (R) } \\
\end{array}$ \\
\hline hasAggregateOfFirstColumn & $\begin{array}{l}\text { D: Each Matrix has exactly } 1 \text { aggregate of weights in its first column } \\
\text { R: ComparisonMatrix hasAggregateOfFirstColumn exactly } 1 \text { double }\end{array}$ \\
\hline hasAggregateOfSecondColumn & $\begin{array}{l}\text { D: Each Matrix has exactly } 1 \text { aggregate of weights in its second column } \\
\text { R: ComparisonMatrix hasAggregateOfSecondColumn exactly } 1 \text { double }\end{array}$ \\
\hline hasAggregateOfThirdColumn & $\begin{array}{l}\text { D: Each Matrix has exactly } 1 \text { aggregate of weights in its third column } \\
\text { R: ComparisonMatrix hasAggregateOfThirdColumn exactly } 1 \text { double }\end{array}$ \\
\hline hasFirstRowPriority & $\begin{array}{l}\text { D: Each Matrix has exactly } 1 \text { priority (a temporary priority inside a } \\
\text { matrix) for the first term of those comparison pairs in its first row. } \\
\text { R: ComparisonMatrix hasFirstRowPriority exactly } 1 \text { double }\end{array}$ \\
\hline hasSecondRowPriority & $\begin{array}{l}\text { D: Each Matrix has exactly } 1 \text { priority (a temporary priority inside a } \\
\text { matrix) for the first term of those comparison pairs in its second row. } \\
\text { R: ComparisonMatrix hasSecondRowPriority exactly } 1 \text { double }\end{array}$ \\
\hline hasThirdRowPriority & $\begin{array}{l}\text { D: Each Matrix has exactly } 1 \text { priority (a temporary priority inside a } \\
\text { matrix) for the first term of those comparison pairs in its third row. } \\
\text { R: ComparisonMatrix hasThirdRowPriority exactly } 1 \text { double }\end{array}$ \\
\hline hasPrincipalEigenvalue & $\begin{array}{l}\text { D: Each Matrix has exactly } 1 \text { Principal Eigenvalue } \\
\text { R: ComparisonMatrix hasPrincipalEigenvalue exactly } 1 \text { double }\end{array}$ \\
\hline hasConsistencyIndex & $\begin{array}{l}\text { D: Each Matrix has exactly } 1 \text { Consistency Index } \\
\text { R: ComparisonMatrix hasConsistencyIndex exactly } 1 \text { double }\end{array}$ \\
\hline hasConsistencyRatio & $\begin{array}{l}\text { D Each Matrix has exactly } 1 \text { Consistency Ratio } \\
\text { R: ComparisonMatrix hasConsistencyRatio exactly } 1 \text { double }\end{array}$ \\
\hline isConsistent & $\begin{array}{l}\text { D: Each Matrix has a boolean value to tell whether it is consistent or not } \\
\mathrm{R} \text { : ComparisonMatrix isConsistent exactly } 1 \text { boolean }\end{array}$ \\
\hline
\end{tabular}


Taking into account the concepts and their relationships in the AHP Ontology and following the rule syntax that are proposed in Jena ${ }^{5}$, some rules that correspond to each sub-step of the step (4) and (5) in the AHP method are created. An example rule is presented in Fig. 4, which is related to the sub-step "a" and to the sub-step "e" in the step (4). The first part of the example rule sums up of all the weights in the first column of a $3 \times 3$ matrix, and the second part shows how to use the priority assessment results to suggest an alternative that has the top priority.

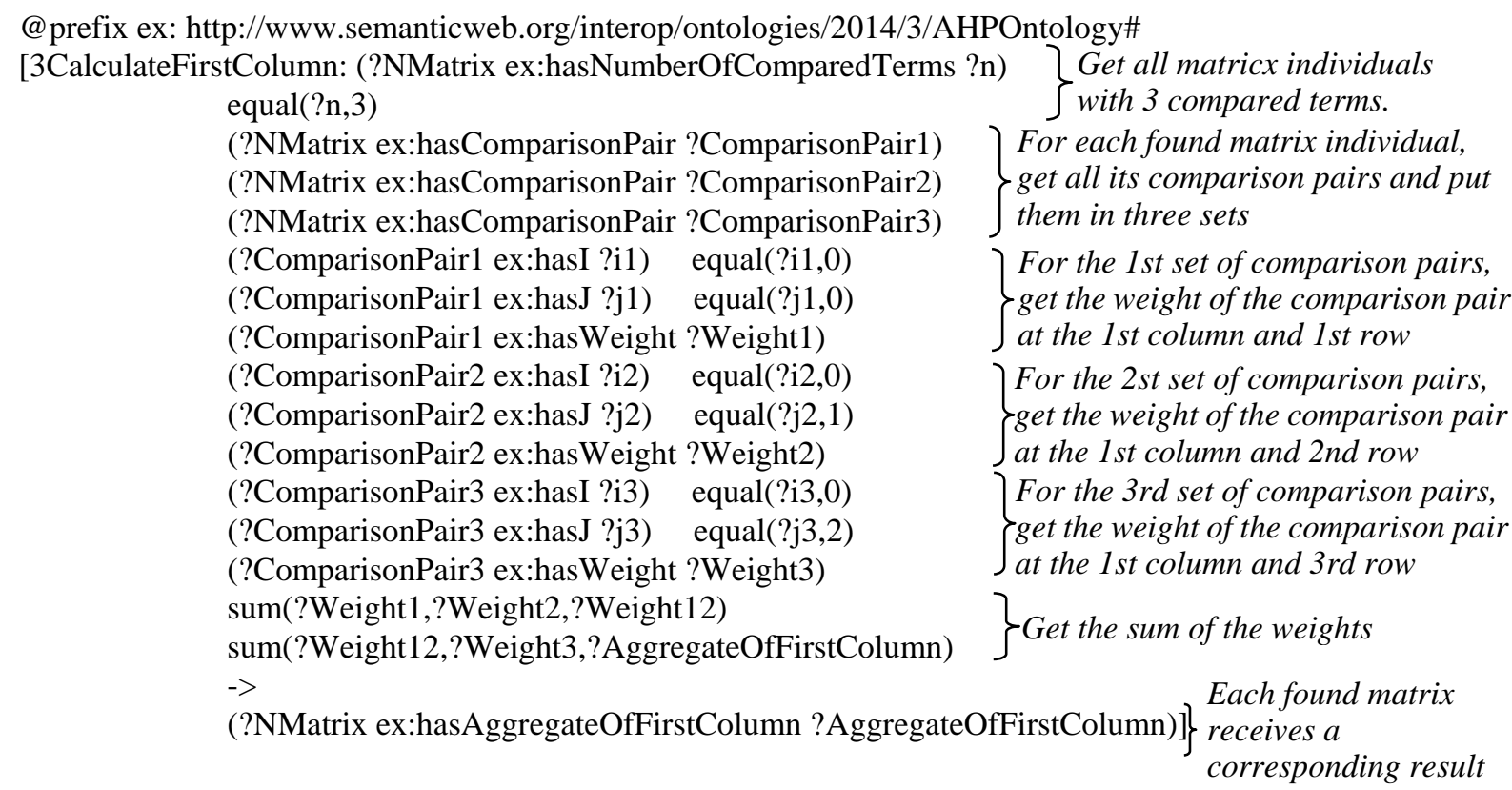

…... $\}$ We omit the rest of the calculations in this example

[3GetTopPriorityAlternative: (?Decision rdf:type ex:Decision) \} Get all decision individuals.

(?Decision ex:hasAlternative ?Alternative1)

(?Decision ex:hasAlternative ?Alternative2)

(?Decision ex:hasAlternative ?Alternative3)

For each found decision individual, get all its alternatives and put them in three sets $\left.\begin{array}{l}\text { notEqual(?Alternative1,?Alternative2) } \\ \text { notEqual(?Alternative2,?Alternative3) }\end{array}\right\}$ Make these sets different from each other (?Alternative1 ex:hasFinalPriority ?FPAlternative1)

(?Alternative2 ex:hasFinalPriority ?FPAlternative2) $\}$ Get the final priority for each alternative (?Alternative3 ex:hasFinalPriority ?FPAlternative3) (?FPAlternative1 ex:hasValue ?Value1) (?FPAlternative2 ex:hasValue ?Value2) Get the Value for each final priority (?FPAlternative3 ex:hasValue ?Value3) lessThan(?Value1,?Value2) lessThan(?Value2,?Value3) $\}$ Get the alternative that has top final priority $\rightarrow \quad$ Suggest the top priority alternative to (?Decision ex:hasSuggestedAlternative ?Alternative3)] $\} \begin{aligned} & \text { Suggest the top priority alter } \\ & \text { its corresponding decision }\end{aligned}$

Fig. 4. Part of the Implementation of Jena Reasoning Rules for a $3 \times 3$ Matrix

\section{The Prototype Tool based on the AHP Ontology}

In order to show how the AHP Ontology with its rules can be applied in reality, we prototyped a priority assessment tool, which is partially based on the framework that we proposed in our previous research [10]. As shown on the left side of Fig. 6, the assessment framework contains four main modules. The Protégé ${ }^{6}$ environment is employed as an ontology editor and browser, which is used to

\footnotetext{
${ }^{5}$ Jena Rule Syntax: http://jena.apache.org/documentation/inference

${ }^{6}$ Protégé: http://protege.stanford.edu/
} 
represent the AHP method into the AHP Ontology with its rules. The AHP Ontology with its rules are saved in the Knowledge Cloud repository. The Jena Reasoner is employed to perform priority assessment and consistency evaluation. The priority assessment tool acts as an intermediate to manage the communications between the users and the other three modules. As can be seen on the right side of Fig. 6, the priority assessment workflow describes how the AHP Ontology with its rules are used in this prototype.

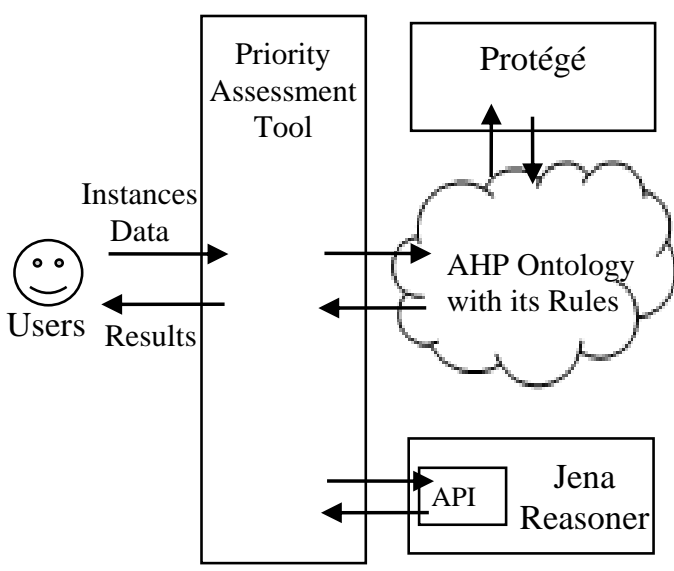

The Assessment Framework

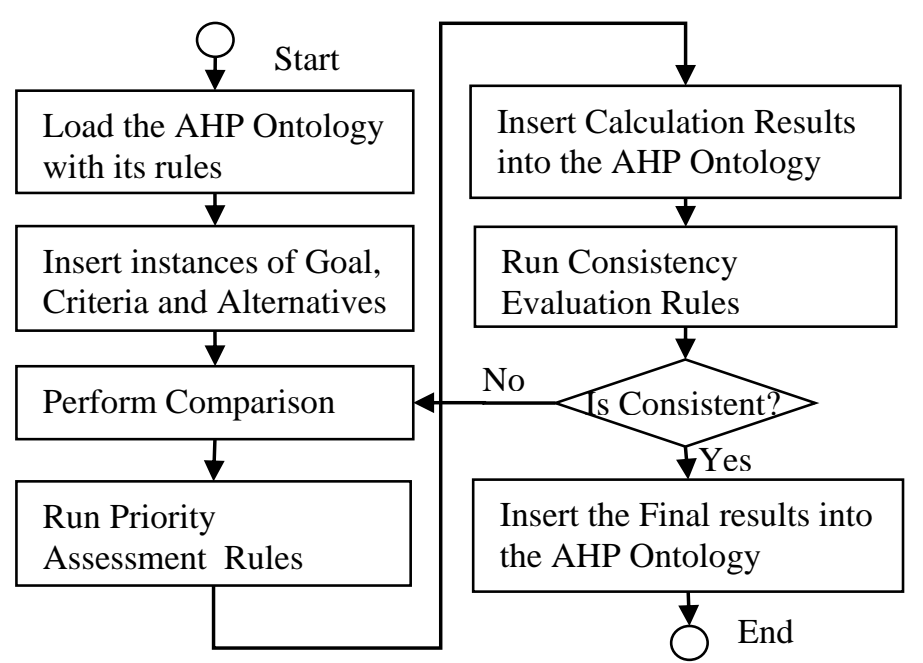

The Assessment Workflow

Fig. 6. The Assessment Framework and the Assessment Workflow

The priority assessment example, which is presented in the thesis [11], is employed as an example to verify this prototype. As it can be seen from Fig. 7, the results that are produced by this prototype shows the AHP Ontology with its rules are capable of performing the priority assessment and consistency evaluation appropriately.

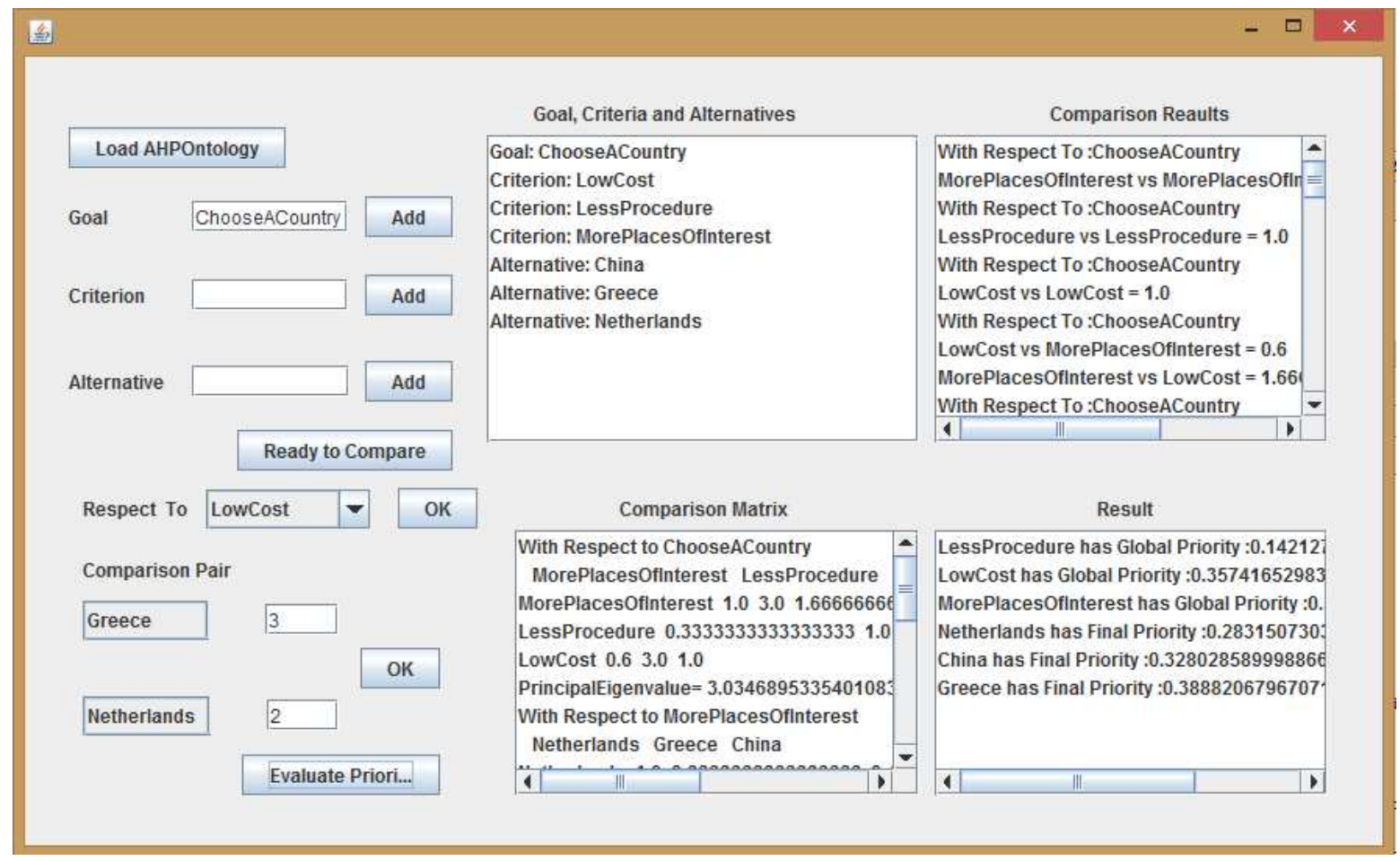

Fig. 7. The Results of Priority Assessment and Consistency Evolution through the Prototype

\section{Conclusions}


In summary, this paper reviews some researches that used ontological representations, which related to the AHP Method, to support their decision-making approaches. Based on the investigation, it identifies three existing drawbacks in current research works. The main contribution of this paper is presenting a novel approach that ontologically represents the AHP Method. It not only offers a way to clearly specify and formalize the concepts, relationships and rules in the AHP Method, but also shows the possibility of obtaining more flexibility and reusability through the ontological representation. Furthermore, with some adjustments of the priority assessment and consistency evaluation rules, we are able to extend the AHP method to adopt different needs. Of course, it should be more interesting, therefore, to ontological represent other kinds of decision-making methods into the knowledge cloud. Our results are encouraging and should be used in a real assessment case. In the next stage, one of our perspective is to apply in the research, which relates to assessment of public administration interoperability [12], to validate the applicability of this study.

\section{Acknowledgements}

The authors would like to thank the financial support provided by the Coordination of Improvement of Higher Education Personal (CAPES) and Pontifícia Universidade Católica do Paraná (PUCPR) in Brazil.

\section{References}

[1] T. L. Saaty: A scaling method for priorities in hierarchical structures. J. Math. Psychol Vol. 15, No. 3 (1977), pp. 234-281

[2] O. S. Vaidya and S. Kumar: Analytic hierarchy process: An overview of applications. Eur. J. Oper. Res. Vol. 169, No. 1 (2006), pp. 1-29

[3] E. Kornyshova and R. Deneckère: Decision-Making Ontology for Information System Engineering. in ER'10 Proceedings of the 29th international conference on Conceptual Modeling, Vancouver, Canada (2010)

[4] V. X. Tran, H. Tsuji, and R. Masuda: A new QoS ontology and its QoS-based ranking algorithm for Web services. Simul. Model. Pract. Theory Vol. 17, No. 8 (2009), pp. 1378-1398

[5] K. Wasielewska, M. Ganzha, M. Paprzycki, P. Szmeja, M. Drozdowicz, I. Lirkov, and C. adica: Applying Saaty's Multicriterial Decision Making Approach in Grid Resource Management. Inf. Technol. Control Vol. 43, No. 1 (2014), pp. 73-87,

[6] T. L. Saaty: How to make a decision: The analytic hierarchy process. Eur. J. Oper. Res. Vol. 48, No. 1 (1990), pp. 9-26

[7] T. L. Saaty: Decision making with the analytic hierarchy process. Int. J. Serv. Sci. Vol. 1, no. 1 (2008), pp. 83-98

[8] T. L. Saaty: Decision making for leaders: the analytic hierarchy process for decisions in a complex world. (RWS Publications, Pittsburgh, 2008)

[9] N. Noy and D. McGuinness: Ontology development 101: A guide to creating your first ontology. Technical Report, Stanford Knowledge Systems Laboratory (2001)

[10] Y. Liao, M. Lezoche, E. R. Loures, H. Panetto, and N. Boudjlida: Formal Semantic Annotations for Models Interoperability in a PLM environment. in The 19th World Congress of the International Federation of Automatic Control (IFAC 2014), Cape Town, South Africa, (2014)

[11] F. Song: Contribution of ontology alignment to enterprise interoperability. University of Bordeaux 1, PhD thesis (2013)

[12] J. M. Cestari, M. Lezoche, E. R. Loures, and H. Panetto: A Research Strategy for Public Administration Interoperability Assessment. in 4th International Conference on Information Society and Technology (ICIST 2014), Kopaonik, Serbia, (2014). 